used as a precision measure. The model was internally validated with bootstrap samples to correct overfitting.

Results: The median follow-up times was 19.4 months (range, 0.77-106.13 months), and the 3-year OS was 41.8\% ( $95 \%$ confidence interval $[\mathrm{CI}]=29.9 \%-58.3 \%$ ). We found International Federation of Gynecology and Obstetrics (FIGO) stage, and adjuvant chemotherapy were independent factors for OS. The $\mathrm{CP}$ of the nomogram integrating with body mass index (BMI), FIGO stage and adjuvant chemotherapy was 0.72 $(95 \% \mathrm{CI}=0.70-0.75)$. The calibration curves for probability of 3-year OS also demonstrated agreement between nomogram prediction and observation data.

Conclusion: The nomogram to predict 3-year OS of UCSs after surgery with simple parameters include BMI, FIGO stage, and adjuvant chemotherapy was implemented in a nomogram and provides accurate prediction of individual patients' prognosis useful for patient counselling and deciding on follow-up strategies.

Poster (E21)

Endometrial Hyperplasia, Endometrial Intra-epithelial Neoplasia, and Endometrial Cancer

https://doi.org/10.3802/jgo.2021.32.S1.E21

\section{A validation study of a modified TCGA classification for patients with endometrial cancer treated with radical surgery and adjuvant chemotherapy}

\footnotetext{
Hiroyuki Yamazaki, ${ }^{1,}$ Kanako Hatanaka, ${ }^{2}$ Hiroshi Asano, Matsuno Yoshihiro, ${ }^{3}$ Yutaka Hatanaka, ${ }^{2}$ Hidemichi Watari

'Department of Obstetrics and Gynecology, Graduate School of Medicine and Faculty of Medicine, Hokkaido University, Sapporo, Japan (cvm5396@elms.hokudai.ac.jp)

${ }^{2}$ Center for Development of Advanced Diagnostics, Research Division of Genome Companion Diagnostics, Hokkaido University Hospital, Sapporo, Japan

${ }^{3}$ Department of Surgical Pathology, Hokkaido University Hospital, Sapporo, Japan
}

Objective: The Cancer Genome Atlas (TCGA) molecular classification for endometrial cancer is expected to propose the most appropriate treatment strategy for each patient. However, the prognostic stratifications remain unclear when adjuvant chemotherapy is applied. This study aimed to validate a modified TCGA classification, ProMisE, for patients who underwent adjuvant chemotherapy at intermediate or high risk of recurrence.

Methods: From 2003 to 2015, the patients who underwent systematic lymphadenectomy were enrolled. The patients were classified by ProMisE using immunohistochemistry for mismatch repair-related molecules (MLH1, MSH2, MSH6, PMS2) and p53, and direct sequencing for hotspot mutations in POLE (exon 9, 13, and 14). The 5-year disease-specific survival rates (5y-DSS) were estimated by the Kaplan-Meier method. Results: A total of 182 patients were analyzed. The median age and follow-up period were 57.4-year-old and 105 months. The patients categorized to stage I, II, III, and IV were 97, 23, 51, and 11, respectively. One hundred twenty-two patients were diagnosed as endometrioid carcinoma (grade 1-2), and 60 were high-grade types, including endometrioid (grade 3), serous, and clear cell carcinomas. Based on ProMisE, 57 patients were categorized into mismatch-repair deficiency (MMR-d), 45 into POLE-EDM (exonuclease domain mutations), 15 into p53 wildtype, and 4 into p53 abnormal. The 5y-DSS was $92.9 \%, 100 \%$, $91.1 \%$, and $75.0 \%$, respectively.

Conclusion: We conclude that ProMisE can stratify the prognosis even when adjuvant chemotherapy was applied for intermediate- and high-risk for recurrence. The 5y-DSS of MMR-d patients seemed better than previous reports, partly because adjuvant chemotherapy could improve their prognosis.

Poster (E22)

Endometrial Hyperplasia, Endometrial Intra-epithelial Neoplasia, and Endometrial Cancer https://doi.org/10.3802/jgo.2021.32.S1.E22

\section{Association between high body mass index and endometrial pathology in premenopausal women with abnormal uterine bleeding}

\author{
Ruangsak Lertkhachonsuk," Nuttida Mahakit, \\ Woranit Apikulprapa, Patou Tantbairojn \\ Chulalongkorn University, Bangkok, Thailand (ruangsak@chula.md)
}

Objective: To find out the correlation between body mass index and endometrial pathology in premenopausal women with abnormal uterine bleeding.

Methods: A cross-sectional study was conducted in King Chulalongkorn Memorial Hospital during 1 January to 31 December 2019. All cases of abnormal uterine bleeding in premenopausal women who had endometrial pathology were recruited. All endometrial histopathology had been reviewed. Patient clinical profiles were recorded. Body mass index (BMI) was calculated by using weight in kilogram divided by square of height in meters. Then all cases were categorized by Asia Pacific BMI classification into underweight $\left(<18.5 \mathrm{~kg} / \mathrm{m}^{2}\right)$, normal weight (18.5-22.9 kg/m²), overweight (23-24.9 kg/m²) and obese $\left(>25 \mathrm{~kg} / \mathrm{m}^{2}\right)$. The pathological reports were classified into 3 groups: normal epithelium, endometrial hyperplasia, and endometrium cancer. Statistical analysis was done SPSS version 